\section{SOME OPEN QUESTIONS CONCERNING BIOLOGICAL GROWTH}

\author{
Carlos Escudero \\ ICMAT (CSIC-UAM-UC3M-UCM), Departamento de Matemáticas, \\ Facultad de Ciencias, Universidad Autónoma de Madrid, Ciudad \\ Universitaria de Cantoblanco, 28049 Madrid, Spain
}

RESUMEN: En este trabajo revisamos brevemente las propiedades de las superficies que crecen de forma radial y su conexión con el crecimiento biológico. Nos vamos a concentrar en modelos simplificados que resultan de la abstracción de sólo considerar el crecimiento del dominio y no la curvatura de la interfaz. Las ecuaciones lineales se pueden resolver exactamente y la fenomenología del crecimiento puede ser inferida de las soluciones explítas. Las ecuaciones no lineales dan lugar a interesantes problemas abiertos que vamos a resumir aquí.

PALABRAS CLAVE: Morfogénesis, Teoría de la Probabilidad, Mecánica Estadística de No Equilibrio, Ecuaciones en Derivadas Parciales Estocásticas.

\section{INTRODUCTION}

Since the origins of the interdisciplinary exchange among mathematics and biology, the development of the form and structure of living organisms has always been considered a fascinating topic [1] [2]. Already present in these seminal works, the necessity of considering physical and mathematical laws, together with the relevant biological principles, has been increasingly recognized in the scientific literature along the years. The first of these works [1], the book On Growth and Form by Sir D'Arcy Wentworth Thompson, is sometimes regarded as the first biomathematics treatise that has ever been written. Among the large number of contributions that can be found in its pages, the chapter relating to the comparison of related forms is particularly representative. In it Thompson describes how differences in the forms of related animals can be formalized by means of simple mathematical transformations. A canonical example is that of the crocodile skulls; in Figure 1 one can see the classical diagrams by Thompson in which he related the form of the skulls of different species of crocodiles by

\section{ALGUNAS CUESTIONES ABIERTAS RELATIVAS AL CRECIMIENTO BIOLÓGICO}

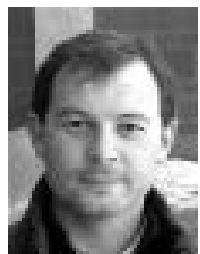

ABSTRACT: We briefly review the properties of radially growing interfaces and their connection to biological growth. We focus on simplified models which result from the abstraction of only considering domain growth and not the interface curvature. Linear equations can be exactly solved and the phenomenology of growth can be inferred from the explicit solutions. Nonlinear equations pose interesting open questions that are summarized herein.

KEY WORDS: Morphogenesis, Probability Theory, Nonequilibrium Statistical Mechanics, Stochastic Partial Differential Equations.

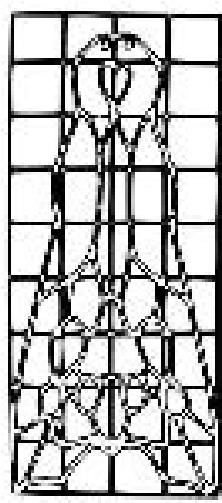

Crocodla aragus

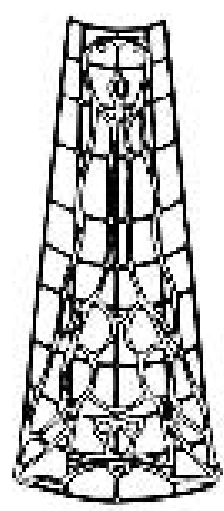

C. gтmitzrus

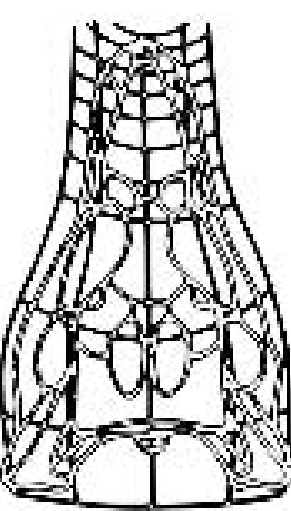

Nologutce ecrosine
Figure 1. Crocodile structure transformations by D'Arcy W. Thompson. This figure shows how simple mathematical transformations can connect the form of the skulls of different species of crocodiles.

means of simple geometric transformations. In his search for a mathematical description of biological forms he also found the remarkable connection among phyllotaxis and the Fibonacci sequence. 
The second seminal work on biomathematics we have mentioned is the article The Chemical Basis of Morphogenesis by Alan Turing [2]. In it Turing explores biological pattern formation by means of systems of reaction-diffusion equations. His goal was understanding how a homogeneous state may lose its stability and give rise to a spatial structure. This is actually what takes place in morphogenetic processes, by means of which a relatively homogeneous distribution of cells starts to develop the shape of a particular organism. To this end he devised a very clever mechanism able to make the homogeneous state lose its stability purely due to the effect of the diffusion of two or more reagents. This Turing instability along with modelling in terms of reaction-diffusion processes are nowadays considered as one of the most important mathematical tools in the field of theoretical morphogenesis [3].

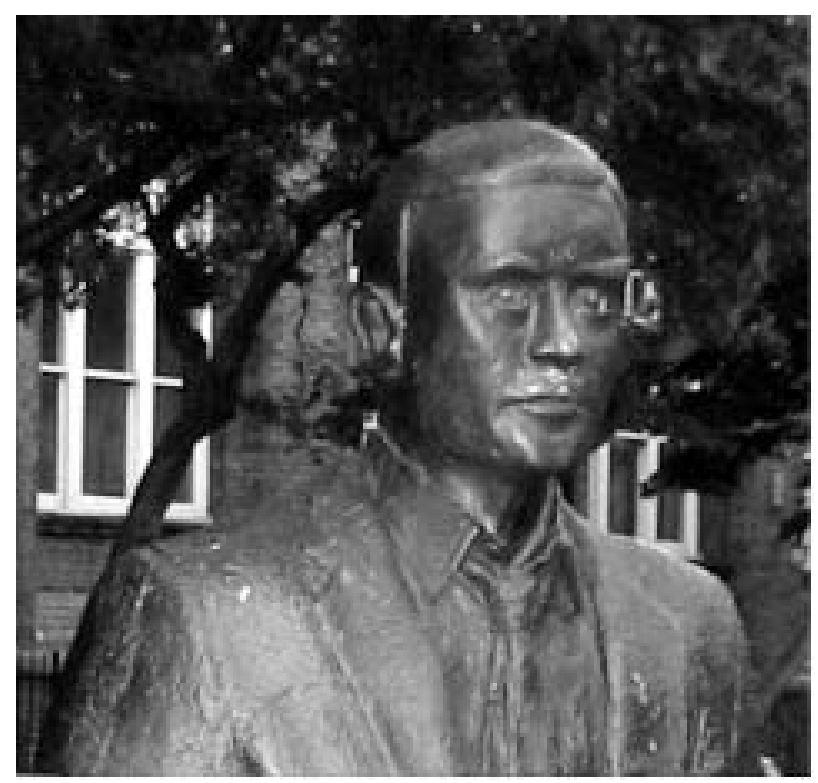

Figure 2. Alan Turing memorial statue in Sackville Park, Manchester, UK (Wikimedia Commons).

Another important example in this context, and object of the present work, is the examination of the properties relating to the architecture of cell colonies, as originally researched by Murray Eden $[4,5]$. The overall appearance of a living organism is conditioned by its genetic expression. Indeed, it escapes to nobody that twins are similar to each other as whole individuals. However, when we regard smaller scale properties of them, like the dermal ridge count of fingerprints or the patterns of retinal venation, their differences are greater even in the case of monozygotic twins. Differences that are expected to grow as our reference spatial scale decreases, and that are particularly evident in the architecture of cell colonies. These and other observations unveil that morphogenetic processes are not completely determined by the genetic expression. Of course, it is widely recognized nowadays that environmental factors play a determinant role in morphogenesis. Both aside and as a consequence of this one would be interested in determining how random factors affect the process of growth, and so how is this process constraint by the laws of probability.

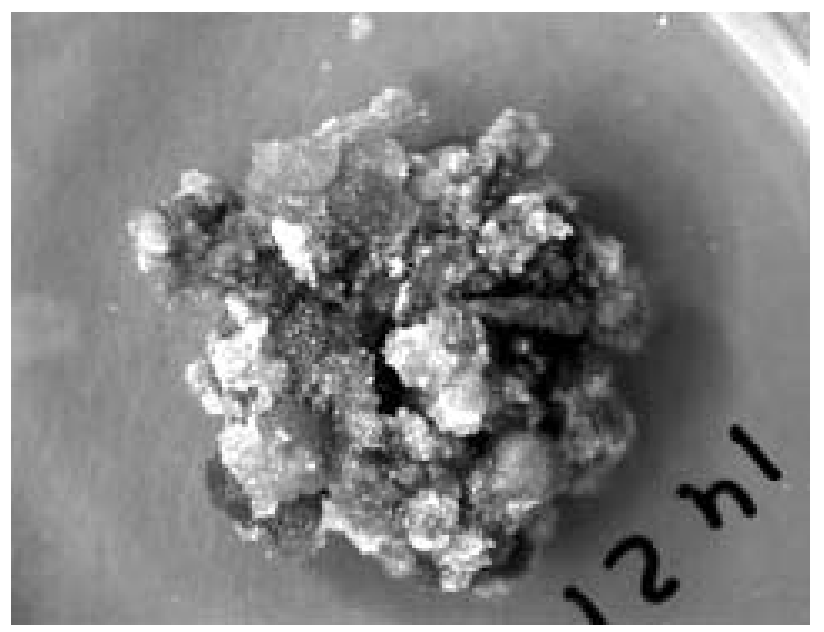

Figure 3. Plant callus: undifferentiated tissue developed on an injured leaf of wild cabbage. Courtesy of the Complex Systems Laboratory, under the leadership of Prof. Javier Galeano at the Universidad Politécnica de Madrid.

The Eden model was introduced in order to shed some light into this question $[4,5]$. It is a simplified model for biological growth, which results from the abstraction of neglecting many of the real aspects of cell colonies development. It concentrates just in the appearance of new cells in the colony periphery; once introduced, cells are never removed from it. This model is to be considered in $\left(\mathbb{Z}^{d}\right)^{\{0,1\}}$, where $d \in \mathbb{N}$ denotes the spatial dimension, and 0 and 1 stand for an empty and an occupied site respectively. Starting from a single cell at the origin, and following a set of probabilistic rules which dictates the frequency and manner in which new cells are introduced in the colony $[4,5]$, a radial form 
develops in the long time. Its macroscopic shape is affected by the underlying lattice structure, and its interface shows fractal properties that are independent of it $[6,7]$. On the applied side, the Eden model and its variants can be used to model some biological processes such as the growth of calli in plants, see Figure 3. On the theoretical side, the Eden model is a type of percolation process, which is known as first passage percolation. Percolation theory is a fascinating branch of probability theory which deals with the properties of connected clusters in random graphs, see Figure 4 , and it is known to pose some of the most changeling questions within this subset of pure mathematics [8].

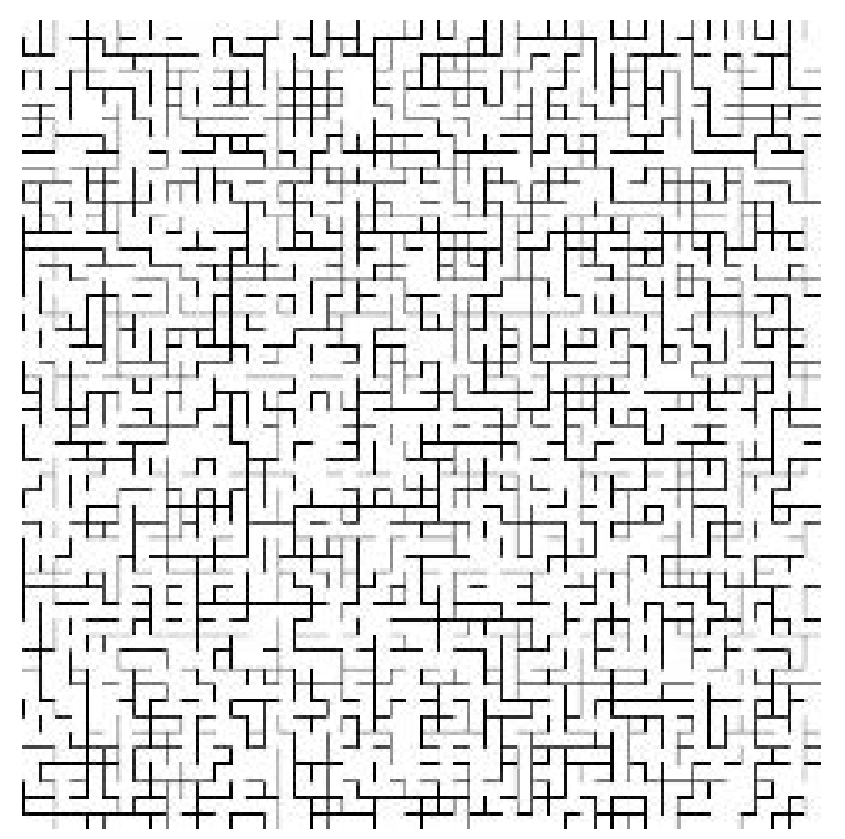

Figure 4. Detail of a bond percolation on the square lattice in two dimensions (Wikimedia Commons).

Although a rigorous connection have never been established, it is widely accepted that the fluctuations of the Eden interface can be described with some suitable stochastic partial differential equation $[6,7,9]$. Such a relation has been proposed in light of numerical results derived with the so called cylindrical Eden model [9]. In this case, for $d=2$, the system is a strip of infinite length and finite width $L$, and the initial condition is a whole semistrip. For large enough $L$ and periodic boundary conditions the system obeys the Family-Vicsek scaling [10], i. e., the twopoints correlation function is of the form

$$
\left\langle h(x, t) h\left(x^{\prime}, t\right)\right\rangle=\left|x-x^{\prime}\right|^{2 \alpha} f\left(\frac{\left|x-x^{\prime}\right|}{t^{1 / 2}}\right),
$$

for short times, where $\langle\cdot\rangle$ is the average over a large number of realizations, $f(\cdot)$ is the scaling function, and $\alpha$ and $z$ are universal quantities known as critical exponents: $\alpha$ is known as the roughness exponent and $z$ as the dynamical exponent, which measures the velocity at which the correlations travel. The function $h(x, t)$ expresses the height of the interface with respect to the initial condition at some given position $x$ and time $t$. The ratio of the critical exponents $\beta=\alpha / z$ constitutes a new exponent measuring the velocity at which the interface width increases during the first stages of growth $[6,7]$. For the dimensionality considered the simulations have measured $\alpha \approx 1 / 2$ and $z \approx 3 / 2$, which place this discrete model in the same universality class as the continuum equation

$$
\partial_{t} h=\nu \nabla^{2} h+\frac{\lambda}{2}(\nabla h)^{2}+F+\xi(x, t)
$$

which is known as the Kardar-Parisi-Zhang (KPZ) equation [11]. In this case $\xi(x, t)$ is a Gaussian distributed spatiotemporal noise delta correlated in both space and time, and $v_{1} \lambda$ and $F$ are real positive parameters. Of course, the cylindrical Eden model differs from its radial counterpart in two features: the interface is curved and grows laterally. Herein we will consider an abstraction of this problem and focus exclusively on lateral growth. As discrete models are usually placed in the universality classes defined by continuum equations, we will study the dynamics of such equations defined on uniformly growing domains.

\section{LINEAR GROWTH}

In this section we will summarize some of the recent results obtained with linear equations. As they are exactly solvable their dynamical structure can be inferred from explicit expressions.

Our study of the dynamics of stochastic growth equations on growing domains begins with a stochastically forced 
diffusion equation, known as the Edwards-Wilkinson (EW) equation [12] in this context, which reads

$$
\partial_{t} h=D \nabla^{2} h+F+\xi(\mathbf{y}, t),
$$

where $\xi(\mathbf{y}, t)$ is a zero-mean Gaussian white noise which correlation is

$$
\left\langle\xi(\mathbf{y}, t) \xi\left(\mathbf{y}^{\prime}, t^{\prime}\right)\right\rangle=\varepsilon \delta\left(\mathbf{y}-\mathbf{y}^{\prime}\right) \delta\left(t-t^{\prime}\right),
$$

$D$ is the diffusion constant, $F$ the constant rate at which mass enters the interface and $\varepsilon$ the noise intensity, all these parameters being positive real numbers. We start considering the conservation law in integral form

$$
\frac{d}{d t} \int_{S_{t}} h(\mathbf{y}, t) d \mathbf{y}=\int_{S_{t}}[-\nabla \cdot \mathbf{j}+\mathcal{F}(\mathbf{y}, t)] d \mathbf{y},
$$

where $S_{t}$ is the uniformly growing domain, $\mathbf{j}=-D \nabla h$ is the current generated by diffusion, and $\mathcal{F}(\mathbf{y}, t)=F+\xi(\mathbf{y}, t)$ is the EW growth mechanism [13]. By applying the Reynolds transport theorem we find

$$
\frac{d}{d t} \int_{S_{t}} h(\mathbf{y}, t) d \mathbf{y}=\int_{S_{t}}\left[\partial_{t} h+\nabla \cdot(\mathbf{v} h)\right] d \mathbf{y},
$$

where $\mathbf{v}(y, t)$ denotes the flow velocity generated by the growing domain. Valid as it is for any domain, the integral conservation law may be expressed in the local form

$$
\partial_{t} h+\nabla \cdot(\mathbf{v} h)=D \nabla^{2} h+\mathcal{F}(\mathbf{y}, t) .
$$

In this equation we readily identify two new terms, the advection one $\mathbf{v} \cdot \nabla h$, and the dilution $h \nabla \cdot \mathbf{v}$. For every $\mathbf{y} \in S_{t,}$ that has evolved from $y_{0} \in S_{t 0}$, we find $\mathbf{v}(\mathbf{y}, t)=\partial \mathbf{y} / \partial t$. Let us now concentrate in one-dimensional domains and then move to higher dimensions. In this case uniform growth translates into $y=g(t) y_{0}$, where $g(t)$ is a temporal function such that $g\left(t_{0}\right)=1$. This yields $v=y \dot{g} / g$, and thus

$$
\partial_{t} h+\frac{\dot{g}}{g}\left(y \partial_{y} h+h\right)=D \partial_{y}^{2} h+F+\xi(y, t) .
$$

For a one-dimensional domain $(0, L(t))$, with $L(t)=g(t) L_{01}$ we change the spatial coordinate $x=y L_{0} / L(t)$, where $L_{0}=L\left(t_{0}\right)$, in order to map the problem into the interval $\left(0, L_{0}\right)$. This transformation counterbalances advection, and so the resulting equation reads

$$
\frac{\partial h}{\partial t}=\left(\frac{L_{0}}{L(t)}\right)^{2} D \frac{\partial^{2} h}{\partial x^{2}}-\frac{\dot{g}}{g} h+F+\sqrt{\frac{L_{0}}{L(t)}} \xi(x, t),
$$

where we have used the fact that the noise is delta correlated. The dilution term has become $h \nabla \cdot \mathbf{v}=-(\dot{g} / g) h$. Dilution has a transparent physical meaning: as the domain grows the incoming mass becomes distributed in a larger (d-dimensional) area. Now we assume that the growth function adopts the power law form $g(t)=\left(t / t_{0}\right)^{\gamma}$, where the growth index $\gamma \geq 0$, to find

$$
\frac{\partial h}{\partial t}=\left(\frac{t_{0}}{t}\right)^{2 \gamma} D \frac{\partial^{2} h}{\partial x^{2}}-\frac{\gamma}{t} h+F+\left(\frac{t_{0}}{t}\right)^{\gamma / 2} \xi(x, t) .
$$

The growth index $\gamma$ is a new degree of freedom of this problem; it cannot be deduced from the other model parameters, and has to be measured directly from the physical system under study.

Now we move to a more general situation in which we consider an arbitrary diffusion operator of order $\zeta$ and an arbitrary spatial dimension $d$, see Eq. 7. From now on the $d$-dimensional coordinates will be denoted $\mathbf{x} \rightarrow x$ and $y \rightarrow y$ for simplicity. In this case, we can proceed exactly in the same way as in the one-dimensional situation to find, instead of equation (10), the equation

$$
\partial_{t} h=-D\left(\frac{t_{0}}{t}\right)^{\xi \gamma}|\nabla|^{\xi} h-\frac{d \gamma}{t} h+F+\left(\frac{t_{0}}{t}\right)^{d \gamma / 2} \xi(x, t),
$$

where the fractional operator $|\nabla|^{\zeta}$ is to be understood in terms of the Fourier transform. Special values of $\zeta$ yield some of the well known equations in this topic, as the EW equation for $\zeta=2$ [12] and the Mullins-Herring equation for $\zeta=4[14,15]$. There is still another way of deriving an equation similar to equation [11] but in which the dilution term is not present. If we just considered the dilatation transformation $x \rightarrow\left(t / t_{0}\right)^{\gamma} x$ instead of domain growth we would find

$$
\partial_{t} h=-D\left(\frac{t_{0}}{t}\right)^{\xi \gamma}|\nabla|^{\zeta} h+F+\left(\frac{t_{0}}{t}\right)^{d \gamma / 2} \xi(x, t) .
$$

The difference among equations 11 and 12 appears already in the amount of mass arriving at the interface [16]. In 
absence of external sources of mass, i. e. $F=\varepsilon=0$, and for no flux boundary conditions the total mass on the surface is conserved

$$
\int_{0}^{L(t)} \cdots \int_{0}^{L(t)} h(y, t) d y=\int_{0}^{L_{0}} \cdots \int_{0}^{L_{0}} h\left(x, t_{0}\right) d x,
$$

for the dilution dynamics (equation 11). In the no dilution situation corresponding to equation 12 we find

$$
\int_{0}^{L(t)} \cdots \int_{0}^{L(t)} h(y, t) d y=\left(\frac{t}{t_{0}}\right)^{d \gamma} \int_{0}^{L_{0}} \cdots \int_{0}^{L_{0}} h\left(x, t_{0}\right) d x
$$

This second case, as we have already mentioned, is pure dilatation, which implies that not only the space grows, but also the interfacial matter grows at the same rate, in such a way that the average density remains constant. Note that this process of matter dilatation, as well as the spatial growth, are deterministic processes.

We have analyzed both types of dynamics, in the absence and presence of dilution, and found a number of measurable consequences. Temporal dynamics can be studied by means of the temporal auto-correlation

$$
\begin{aligned}
A\left(t, t^{\prime}\right) \equiv & \frac{\left\langle h(x, t) h\left(x, t^{\prime}\right)\right\rangle_{0}}{\left\langle h(x, t)^{2}\right\rangle_{0}^{1 / 2}\left\langle h\left(x, t^{\prime}\right)^{2}\right\rangle_{0}^{1 / 2}} \sim\left(\frac{\min \left\{t, t^{\prime}\right\}}{\max \left\{t, t^{\prime}\right\}}\right)^{\lambda}, \\
& \text { for } \max \left\{t, t^{\prime}\right\} \gg \min \left\{t, t^{\prime}\right\},
\end{aligned}
$$

where $\lambda$ is the auto-correlation exponent and $\langle\cdot\rangle_{0}$ denotes the average with the zeroth mode contribution suppressed. When dilution is considered the auto-correlation exponent takes the form

$$
\lambda=\left\{\begin{array}{lll}
\beta+d / \zeta & \text { if } & \gamma<1 / \zeta, \\
\beta+\gamma d & \text { if } & \gamma>1 / \zeta,
\end{array}\right.
$$

or alternatively

$$
\lambda=\beta+\frac{d}{z_{\lambda}}
$$

where $\beta=1 / 2-d /(2 \zeta)$ and

$$
z_{\lambda}=\min \left\{\zeta_{1} 1 / \gamma\right\}
$$

In absence of dilution we find

$$
\lambda=\left\{\begin{array}{lll}
\frac{1}{2}+\frac{d}{2 \zeta}-d \gamma & \text { if } & \gamma<1 / \zeta, \\
\frac{1}{2}-\frac{d}{2 \zeta} & \text { if } & \gamma>1 / \zeta,
\end{array}\right.
$$

or alternatively

$$
\lambda=\beta+\frac{d}{z_{\text {eff }}}
$$

where $\beta=1 / 2-d /(2 \zeta)$ and

$$
z_{\text {eff }}=\left\{\begin{array}{lll}
\zeta /(1-\gamma \zeta) & \text { if } & \gamma<1 / \zeta, \\
\infty & \text { if } & \gamma>1 / \zeta .
\end{array}\right.
$$

From these formulas one can clearly read that when dilution is suppressed there is no mechanism for correlations propagation. Otherwise dilution is the responsible for the propagation of correlations in the fast growth regime, $\mathrm{i}$. e. when $\gamma>1 / \zeta$.

Complementary information can be obtained from the interface persistence. The persistence of a stochastic process denotes its tendency to continue in its current state. When considering the dynamics of a fluctuating interface, one refers to the persistence probability $P_{+}\left(t_{1}, t_{2}\right)\left(P_{-}\left(t_{1}, t_{2}\right)\right)$ as the pointwise probability that the interface remains above (below) its profile at $t_{1}$ up to time $t_{2}>t_{1}[18,19]$. Herein, as in [17], we concentrate on the case in which the initial profile is flat, and we suppress the contribution coming from the zeroth mode again. For the stochastic differential equations under consideration the symmetry $h_{n} \rightarrow-h_{n}$ for all Fourier modes $n \neq 0$ holds, implying the equality $P_{+}=P_{-} \equiv P$. For long times $t_{2} \gg t_{1}$ we have the power law behavior $[18,19]$

$$
P\left(t_{1}, t_{2}\right) \sim\left(t_{1} / t_{2}\right)^{\theta},
$$

defining the persistence exponent $\theta$. It was previously calculated in the limit $\zeta \rightarrow \infty$ when $\gamma=0$ [19].

$$
\theta \approx \frac{1}{2}+\frac{2 \sqrt{2}-1}{2} \frac{d}{\zeta}
$$

up to higher order terms, and in this same limit when $d=1$ and $\gamma=1$ [17] 


$$
\theta \approx \frac{1}{2}-\frac{1}{2 \zeta}
$$

up to higher order terms and in the absence of dilution. We have calculated the persistence exponent [13], again in the limit $\zeta \rightarrow \infty$ and assuming the inequality $\gamma>1 / \zeta$, in the presence of dilution

$$
\theta \approx \frac{1}{2}+d \gamma-\frac{d}{2 \zeta}
$$

up to higher order terms, and in the absence of it

$$
\theta \approx \frac{1}{2}-\frac{d}{2 \zeta}
$$

generalizing the previous result [17]. The exponent $\theta=1 / 2$ characterizes neutrally persistent interfaces, which are those deprived of a relaxation mechanism (i. e. $D=0$ in equation 12). For $\theta<1 / 2$ the interface is persistent and for $\theta>1 / 2$ it is antipersistent. Note that if dilution acts on the interface then it is antipersistent, as in the case of no domain growth; contrarily, if dilution is not present, the interface becomes persistent.

Before we start calculating spatial correlations let us note that domain growth induces the length scale $\left|x-x^{\prime}\right| \sim t^{(1-\zeta \gamma) / \zeta}$. First we show the scaling form that the two points correlation function adopts for "microscopic" spatial scales $\left|x-x^{\prime}\right| \ll t^{(1-\zeta \gamma) / \zeta}$ in the fast growth regime. In this case one has [16]

$$
\begin{gathered}
\left\langle h(x, t) h\left(x^{\prime}, t\right)\right\rangle-\langle h(x, t)\rangle^{2} \approx \\
\approx\left|x-x^{\prime}\right|^{\zeta-d} t^{\gamma(\zeta-d)} \mathcal{F}\left[\left|x-x^{\prime}\right| t^{(\zeta \gamma-1) / \zeta}\right],
\end{gathered}
$$

or in Lagrangian coordinates $\left|y-y^{\prime}\right|=\left|x-x^{\prime}\right| t^{\gamma}$

$$
\begin{aligned}
& \left\langle h(y, t) h\left(y^{\prime}, t\right)\right\rangle-\langle h(y, t)\rangle^{2} \approx \\
& \approx\left|y-y^{\prime}\right|^{\zeta-d} \mathcal{F}\left[\frac{\left|y-y^{\prime}\right|}{t^{1 / \zeta}}\right],
\end{aligned}
$$

where we have assumed the inequality $\zeta>d$ and the statistical isotropy and homogeneity of the system in the limit in which the scaling form holds. As dilution does not act on such a microscopic scale, these results are independent of whether we contemplate dilution or not. Things are different for macroscopic length scales $\left|x-x^{\prime}\right| \gg t^{(1-\zeta \gamma) / \zeta}$. In this limit dilution has a measurable action, and when it is included in the interface equation of motion the resulting correlation is [13]

$$
\left\langle h(y, t) h\left(y^{\prime}, t\right)\right\rangle-\langle h(y, t)\rangle^{2} \sim t \delta\left(y-y^{\prime}\right),
$$

which is simply the short time limit of equation 28 . If dilution is suppressed we find however $[21,22]$

$$
\left\langle h(y, t) h\left(y^{\prime}, t\right)\right\rangle_{0} \sim \begin{cases}t \delta\left(y-y^{\prime}\right) & \text { if } \gamma<1 / d, \\ t \ln (t) \delta\left(y-y^{\prime}\right) & \text { if } \gamma=1 / d, \quad \text { (30) } \\ t^{\gamma d} \delta\left(y-y^{\prime}\right) & \text { if } \gamma>1 / d .\end{cases}
$$

In this case we see that for fast enough growth memory effects appear and modify the time dependent prefactor $[21,22]$. The increase of this prefactor reflects the mass excess that enters the interface when dilution is not operating as shown in equation 14 . We note that these correlations are modified when one considers actual radial growth due to topological considerations, a fact that has unambiguous effects on the interface scaling [23]. A consequence of all these correlations is the scale dependent fractal dimension

$$
\begin{gathered}
d_{f}\left(\left|x-x^{\prime}\right|, t\right)= \\
\begin{cases}1+(3 d-\zeta) / 2 & \text { if }\left|x-x^{\prime}\right| \ll t^{(1-\zeta \gamma) / \zeta}, \\
d+1 & \text { if }\left|x-x^{\prime}\right| \gg t^{(1-\zeta \gamma) / \zeta},\end{cases}
\end{gathered}
$$

which is independent of whether we contemplate dilution or not. These asymptotic values suggest the self-similar form of the fractal dimension

$$
d_{f}=d_{f}\left(\frac{\left|x-x^{\prime}\right|}{t^{(1-\zeta \gamma) / \zeta}}\right)
$$

which would imply its invariance with respect to the dilatation $x \rightarrow b x_{1} t \rightarrow b^{Z} t_{1}$ and $d_{f} \rightarrow b^{\alpha_{f}} d_{f,}$ for $z_{f}=\zeta \mid$ $(1-\zeta \gamma), \alpha_{\mathrm{f}}=0$ and $\mathrm{b}$ a real number strictly greater than one.

As a final note let us mention that the assumption $\zeta>d$ is fundamental in order to get the correlations specified by equation (28). As we have seen, in this case the dynamical exponent is universal and given by $z=\zeta$. For $\gamma=0$ and $\zeta=d=1$ the dynamical exponent is still universal and given by $z=1$; however for $\gamma=\zeta=d=1$ and in the 
absence of dilution this exponent becomes non-universal and given by $z=F /(D+F) \in(0,1)[20]$. The presence of dilution restores universality and the non-growing domain result $z=1$ [16].

\section{Nonlinear Growth}

The open questions in this topic are related, not surprisingly, to the appearance of nonlinear terms in the corresponding equations of motion. One of the most popular nonlinear models in this context is the KPZ equation, which as we have commented in the Introduction is related to the biologically motivated Eden model. As we will see, understanding the KPZ equation on a growing domain may shed some light on some of the properties of the classical version of this model.

The KPZ equation on a growing domain reads [16]

$$
\begin{aligned}
& \partial_{t} h=\nu\left(\frac{t_{0}}{t}\right)^{2 \gamma} \nabla^{2} h+\frac{\lambda}{2}\left(\frac{t_{0}}{t}\right)^{2 \gamma}(\nabla h)^{2}- \\
& -\frac{d \gamma}{t} h+\gamma F t^{\gamma-1}+\left(\frac{t_{0}}{t}\right)^{d \gamma / 2} \xi(x, t) .
\end{aligned}
$$

Of course, if we just considered the dilatation $x \rightarrow\left(t / t_{0}\right)^{\gamma} x$ we would find

$$
\begin{aligned}
& \qquad \begin{array}{l}
\partial_{t} h=\nu\left(\frac{t_{0}}{t}\right)^{2 \gamma} \nabla^{2} h+\frac{\lambda}{2}\left(\frac{t_{0}}{t}\right)^{2 \gamma}(\nabla h)^{2}+ \\
+\gamma F t^{\gamma-1}+\left(\frac{t_{0}}{t}\right)^{d \gamma / 2} \xi(x, t)
\end{array} \\
& \qquad h(x, t)=\frac{2 \nu}{\lambda} \ln \left\{\frac{(1-2 \gamma)^{d / 2} \exp \left[F \lambda t^{\gamma} /(2 \nu)\right]}{\left[4 \pi t_{0}^{2 \gamma}\left(t^{1-2 \gamma}-t_{0}^{1-2 \gamma}\right)\right]^{d / 2}} \int_{\mathbb{x}^{d}} \exp \right. \\
& \text { for given initial conditions } u\left(x, t_{0}\right) \text { and } h\left(x, t_{0}\right) \text {. It is clear } \\
& \text { by regarding this formula that decorrelation at the deter- } \\
& \text { ministic level will happen for } \gamma>1 / 2 \text {. It is still necessary } \\
& \text { to find out if at the stochastic level this threshold will be } \\
& \text { moved to } \gamma>2 / 3 \text {. If we consider the dilution KPZ equa- } \\
& \text { tion } 33 \text { then transforming Hopf-Cole we would find the }
\end{aligned}
$$$$
h(x, t)=\frac{2 \nu}{\lambda} \ln \left\{\frac{(1-2 \gamma)^{d / 2} \exp \left[F \lambda t^{\gamma} /(2 \nu)\right]}{\left[4 \pi t_{0}^{2 \gamma}\left(t^{1-2 \gamma}-t_{0}^{1-2 \gamma}\right)\right]^{d / 2}} \int_{\mathbb{R}^{d}} \exp \left[-\frac{|x-y|^{2}(1-2 \gamma)}{4 t_{0}^{2 \gamma}\left(t^{1-2 \gamma}-t_{0}^{1-2 \gamma}\right)}+\frac{\lambda}{2 \nu} h\left(y, t_{0}\right)\right] d y\right\},
$$
nonlinear equation
As we have shown in the previous section, the dilution mechanism fixes the Family-Vicsek scaling in the fast growth regime. In the radial Eden model case, assuming it belongs to the KPZ universality class, we would have $z=3 / 2$ in $d=1$ and $\gamma=1$. And so, one would naïfly expect that the resulting interface is uncorrelated and we have to resort on dilution effects in order to fix the Family-Vicsek scaling and get rid of memory effects. But here comes the paradoxical situation. There are two main symmetries associated with the d-dimensional KPZ equation: the HopfCole transformation which maps it onto the noisy diffusion equation [24] and Galilean invariance which have been traditionally related to the non-renormalization of the KPZ vertex at an arbitrary order in the perturbation expansion [25]. In the case of the no-dilution KPZ equation 34 both symmetries are still present. Indeed, this equation transforms under the Hopf-Cole transformation $u=\exp [\lambda$ $h /(2 v)]$ to

$$
\partial_{t} u=\nu\left(\frac{t_{0}}{t}\right)^{2 \gamma} \nabla^{2} u+\frac{\gamma F \lambda}{2 \nu} t^{\gamma-1} u+\frac{\lambda}{2 \nu}\left(\frac{t_{0}}{t}\right)^{d \gamma / 2} \xi(x, t) u,
$$

which is again a noisy diffusion equation and it can be explicitly solved in the deterministic limit $\varepsilon=0$. We find in this case

$$
\begin{gathered}
u(x, t)=\frac{(1-2 \gamma)^{d / 2} \exp \left[F \lambda t^{\gamma} /(2 \nu)\right]}{\left[4 \pi t_{0}^{2 \gamma}\left(t^{1-2 \gamma}-t_{0}^{1-2 \gamma}\right)\right]^{d / 2}} \\
\int_{\mathbb{R} d} \exp \left[-\frac{|x-y|^{2}(1-2 \gamma)}{4 t_{0}^{2 \gamma}\left(t^{1-2 \gamma}-t_{0}^{1-2 \gamma}\right)}\right] u\left(y, t_{0}\right) d y,
\end{gathered}
$$

which corresponds to

$$
\begin{aligned}
& \partial_{t} u=\nu\left(\frac{t_{0}}{t}\right)^{2 \gamma} \nabla^{2} u-\frac{d \gamma}{t} u \ln (u)+ \\
& +\frac{\gamma F \lambda}{2 \nu} t^{\gamma-1} u+\frac{\lambda}{2 \nu}\left(\frac{t_{0}}{t}\right)^{d \gamma / 2} \xi(x, t) u,
\end{aligned}
$$

which may be thought of as a time dependent and spatially distributed version of the Gompertz differential equation [26]. In this case it is not evident how to find an 
explicit solution at the deterministic level and what would be its decorrelation threshold.

Galilean invariance means that the transformation

$$
x \rightarrow x-\lambda v t, \quad h \rightarrow h+v x, \quad F \rightarrow F-\frac{\lambda}{2} v^{2},
$$

where $v$ is an arbitrary constant vector field, leaves the KPZ equation invariant. In case of no dilution this transformation can be replaced by

$$
\begin{gathered}
x \rightarrow x-\frac{\lambda}{1-2 \gamma} v t_{0}^{2 \gamma} t^{1-2 \gamma}, \quad h \rightarrow h+v x, \\
F \rightarrow F-\frac{\lambda}{2 \gamma} v^{2} t_{0}^{2 \gamma} t^{1-3 \gamma},
\end{gathered}
$$

which leaves invariant equation 34. If we consider dilution, then it is not clear how to extend this transformation to leave equation 33 invariant. The main difficulty comes from the dilution term which yields a non-homogeneous contribution to the dynamics as a response to the transformation $h \rightarrow h+v x$. So in summary we may talk of a certain sort of Galilean invariance which is obeyed by the no-dilution KPZ dynamics (equation 34) and is lost when dilution is taken into account. If it were found that the dilution equation 33 obeys the traditional KPZ scaling (at least in some suitable limit), then that would put into question the role that Galilean invariance has in fixing the exponents. The KPZ critical exponents are believed to obey the scaling relation $\alpha+z=2$ in all spatial dimensions, a relation that has been traditionally attributed to Galilean invariance, although this interpretation has been recently put into question [27-31].

There is still another fundamental symmetry of the KPZ equation, but this time it just manifests itself in one spatial dimension: the so called fluctuation-dissipation theorem $[6,7]$. It basically says that for long times, when saturation has already being achieved, the nonlinearity ceases to be operative and the resulting interface profile would be statistically indistinguishable from that created by the EW equation. For fast domain growth, we know from the linear theory that the interface never becomes correlated, and it operates, in this sense, as if it were effectively in the short time regime for all times [13]. As a consequence, the fluctuation-dissipation theorem is not expected to play any role in this case. Of course, this result would be independent of whether we contemplated dilution or not.

\section{DISORDER}

It is quite natural to consider propagation into disordered media in the context of biological growth. One could, for instance, imagine the development of a bacterial infection inside a host body. This is, of course, the propagation of a bacterial front inside a medium with a extremely low degree of symmetry.

Apart from the classical KPZ equation which is driven by thermal noise, a different version in which this noise is replaced by quenched disorder has been considered in the literature $[6,7]$

$$
\begin{gathered}
\partial_{t} h=\nu \nabla^{2} h+\frac{\lambda}{2}(\nabla h)^{2}+F+\xi(x, h), \\
\langle\xi(x, h)\rangle=0, \\
\left\langle\xi(x, h) \xi\left(x^{\prime}, h^{\prime}\right)\right\rangle=\varepsilon \delta\left(x-x^{\prime}\right) \delta\left(h-h^{\prime}\right),
\end{gathered}
$$

which is nonlinear even when $\lambda=0$. All the problems considered in this section are open in this simpler case as well. A way of understanding this equation is considering the simplified random deposition version of it

$$
\partial_{t} h=F+\xi(x, h)
$$

which is actually an ordinary differential equation in which the position $x$ acts just as a parameter, at least if the quenched disorder is conveniently regularized. And thus, let us consider the auxiliary problem

$$
\begin{gathered}
\frac{d h}{d t}=F+\eta(h), \\
\langle\eta(h)\rangle=0, \\
\left\langle\eta(h) \eta\left(h^{\prime}\right)\right\rangle=\varepsilon \delta\left(h-h^{\prime}\right),
\end{gathered}
$$

It is similar to the stochastic problem

$$
\begin{gathered}
\frac{d h}{d t}=F+\eta(t), \\
\langle\eta(t)\rangle=0, \\
\left\langle\eta(t) \eta\left(t^{\prime}\right)\right\rangle=\varepsilon \delta\left(t-t^{\prime}\right),
\end{gathered}
$$


which solution is

$$
h(t)=h\left(t_{0}\right)+F\left(t-t_{0}\right)+\sqrt{\varepsilon} W\left(t-t_{0}\right),
$$

where $t_{0}$ is the initial time and $W(t)$ is a Wiener process, so we basically have two superposed motions: constant drift and Brownian motion. Classical Brownian motion describes a particle choosing its direction of motion randomly every time step. If the noise is position dependent, i. e. $\eta=\eta(h)$, then the direction of motion is already prescribed in every spatial point. For a discrete version of this process, say an unbiased random walk on $\mathbb{Z}$ for which the jump direction is specified in every site, if the system revisits any location, then it is trapped forever (jumping forth and back in the last two visited sites). The only way to prevent (this of other sort of) trapping is to consider a sufficiently large $F_{\text {, }}$ so the system is evolving over new positions all of the time. Something similar happens in equation 41: for values of $F$ smaller than a critical one $F_{c}$ the interface becomes pinned, while for larger values the interface propagates [6].

It would be interesting to analyze the interplay of spatial disorder with a growing domain size. We again have two possibilities, the equation with dilution

$$
\begin{aligned}
& \partial_{t} h=\nu\left(\frac{t_{0}}{t}\right)^{2 \gamma} \nabla^{2} h+\frac{\lambda}{2}\left(\frac{t_{0}}{t}\right)^{2 \gamma}(\nabla h)^{2}- \\
& -\frac{d \gamma}{t} h+\gamma F t^{\gamma-1}+\left(\frac{t_{0}}{t}\right)^{d \gamma / 2} \xi(x, h),
\end{aligned}
$$

and the one without it

$$
\begin{aligned}
\partial_{t} h & =\nu\left(\frac{t_{0}}{t}\right)^{2 \gamma} \nabla^{2} h+\frac{\lambda}{2}\left(\frac{t_{0}}{t}\right)^{2 \gamma}(\nabla h)^{2}+ \\
& +\gamma F t^{\gamma-1}+\left(\frac{t_{0}}{t}\right)^{d \gamma / 2} \xi(x, h) .
\end{aligned}
$$

Note that, contrary to the classical case in which the interface propagates linearly in time, in this case the velocity of propagation would be $\sim t^{\gamma}$. It would be interesting to clarify whether $\gamma=1$ plays a critical role in the dynamics or not. In other words, whether the interface is always pinned for $\gamma<1$ and always moving for $\gamma>1$, or if there is a dependence on the parameters values in these cases too. If the second situation held, it would be yet necessary to clarify whether there are other possible critical values for $\gamma$. According to the microscopic description commented in this section, it seems plausible that $\gamma=1$ is indeed the critical value of the growth index separating pinned and unpinned regimes.
Even when $\gamma=1$ there is an interesting question associated with dilution. We know that dilution keeps constant the amount of matter on the interface, while suppressing it we get a mass excess. For linearly in time growing interfaces we know that the value of $F$, which describes the amount of matter arriving at the interface, controls the possibility of interface pinning/unpinning. If no dilution is present, the mass excess could act as effectively increasing the value of $F$, and thus facilitating interface unpinning. It would be interesting to quantitatively determine how much the threshold of pinning is moved in the absence of dilution, if this is indeed the case.

\section{Summary and Conclusions}

As we have seen, a consequence of the linear theory is that dilution erases the memory effects and this way restores the classical Family-Vicsek scaling [21, 22]. Otherwise, for fast domain growth, a series of unexpected consequences arise, as the modification of the random deposition correlation, the lost of the antipersistent character of the fluctuating interface and even the appearance of nonuniversal critical exponents. In this respect, dilution can be thought of as the mechanism which maintains some of the most characteristic features of surface growth when we let the domain size grow in time.

On the other hand, some of the well known symmetries of the KPZ equation, as Galilean invariance and mapping to the directed polymer problem, are maintained in a dilating setting but lost by virtue of dilution. So, in principle, one would expect that in the absence of dilution memory effects could be present in the KPZ dynamics, and this way some its characteristic features would be lost. However, it is somehow paradoxical that it is exactly this absence of dilution what maintains the classical symmetries of this equation. Furthermore, these symmetries have been sometimes considered as necessary ingredients in the resulting KPZ scaling. If we found that the $\mathrm{KPZ}$ equation in a rapidly growing domain and in presence of dilution behaved in a similar way to its classical counterpart, that would suggest that the symmetries present in the standard situation are not playing such a necessary role.

As we have already mentioned, the motivation for studying radial growth models such as the Eden or different 
ones partially comes from the possible similarity of these with some forms of biological development, such as for instance bacterial colonies formation. The results of our study can be translated into this context to obtain some simple conclusions, provided the modelling assumptions make sense for some biological system. The structure of a rapidly developing bacterial colony would be dominated by dilution effects, originated in the birth of new cells which volume causes the displacement of the existent cells. If the rate of growth is large enough this motion will dominate over any possible random dispersal of the bacteria. It is remarkable that such a consequence simply appears by considering domain growth, while it is not necessary to introduce corrections coming from the finite size of the constituents. This is the dilution dominated situation we have formalized by means of the (decorrelation) inequality $\gamma>1 / \zeta$. If we were to introduce some control protocol in order to keep the consequences of bacterial propagation to a minimum we would need to eliminate colony constituents (possibly randomly selected) at a high enough rate so the effective growth velocity were one that reversed the decorrelation inequality. For the two dimensional radial Eden model, accepting it belongs to the KPZ universality class, one finds $\gamma=1$ and $z=3 / 2$. If $z$ played the same role for the nonlinear $\mathrm{KPZ}$ equation as $\zeta$ for the linear equations considered herein (as it is reasonable to expect), the Eden model would be in the dilution dominated regime. In order to control it we would need to eliminate its cells at a rate such that the effective growth index obeyed $\gamma<2 / 3$. For the three dimensional Eden model, if its behavior were still analogous to that of the KPZ equation, we would find $z>3 / 2$ and thus a greater difficulty for control. Note that for the particular growth rules of the Eden model one would need to eliminate peripheral cells in order to control the system. This would not be so in the case of an actual bacterial colony, for which bulk cells are still able to reproduce, and so cell elimination could be performed randomly across the whole colony. Of course, these conclusions are speculative as long as stochastic growth equations are not proved to reasonably model some biological system. With respect to the problem of the experimental verification of the theoretical results, one would be interested in finding a method for measuring the exponents. These could perhaps be measured using the long time dependence of the interface variance on the initial system size and time

$$
\begin{gathered}
\left\langle h(x, t)^{2}\right\rangle-\langle h(x, t)\rangle^{2} \\
\sim\left\{\begin{array}{lll}
L_{0}^{2 \alpha} t^{2 \beta} \sim L(t)^{2 \alpha} t^{2 \beta-2 \gamma \alpha} & \text { for } & \gamma>1 / z \\
L_{0}^{2 \alpha} t^{2 \gamma \alpha} \sim L(t)^{2 \alpha} & \text { for } & \gamma<1 / z
\end{array}\right.
\end{gathered}
$$

that we found for both the dilution and no-dilution linear dynamics in [13]. This way one could in principle experimentally determine both exponents $\alpha$ and $\beta$ and as a consequence the ratio $z=\alpha \mid \beta$ in the fast growth regime. Subtracting the inverse of the so obtained value for the dynamic exponent $z$ from the measured value of

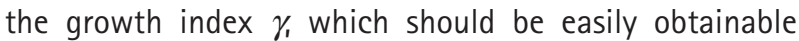
from experiments, one could estimate the distance from correlation, and in turn the possible necessary strength of a control protocol.

\section{ACKNOWLEDGMENTS}

This work has been partially supported by the MICINN (Spain) through Project No. MTM2008-03754.

\section{REFERENCES}

1 D. W. Thompson, On Growth and Form (Cambridge University Press, Cambridge, 1917).

Recibido: 9 de noviembre de 2009 Aceptado: 12 de agosto de 2010
2 A. M. Turing, Phil. Trans. R. Soc. B 237, 37 (1952).
3 J. D. Murray, Mathematical Biology. Springer-Verlag, 3rd ed. in 2 vols.: Mathematical Biology: I. An Introduction, 2002; Mathematical Biology: Il. Spatial Models and Biomedical Applications, 2003.

4 M. Eden, in Symposium on Information Theoryin Biology, edited byH.P.Yockey (Pergamon Press, New York, 1958).

5 M. Eden, in Proceedings of the Fourth Berkeley Symposium on Mathematical Statistics and Probability, edited by J. Neyman (University of California Press, Berkeley, 1961). 
6 A.-L. Barabási and H. E. Stanley, Fractal Concepts in Surface Growth (Cambridge University Press, Cambridge, 1995).

7 T. Halpin-Healy and Y.-C. Zhang, Phys. Rep. 254, 215 (1995).

8 B. Bollobás and 0. Riordan, PercoIation (Cambridge University Press, Cambridge, 2006).

9 J. M. Hammersley and G. Mazzarino, Comb. Probab. Comput. 3, 471 (1994).

10 F. Family and T. Vicsek, J. Phys. A 18, L75 (1985).

11 M. Kardar, G. Parisi, and Y.-C. Zhang, Phys. Rev. Lett. 56, 889 (1986).

12 S. F. Edwards and D. R. Wilkinson, Proc. R. Soc. London Ser. A 381, 17 (1982).
13 C. Escudero, J. Stat. Mech. P07020 (2009).

14 W. W. Mullins, J. Appl. Phys. 28, 333 (1957).

15 C. Herring, J. Appl. Phys. 21, 301 (1950).

16 C. Escudero, arXiv:0909.5304.

17 S. B. Singha, J. Stat. Mech. P08006 (2005).

$18 \mathrm{H}$. Kallabis and J. Krug, Europhys. Lett. 45, 20 (1999).

19 J. Krug, H. Kallabis, S. N. Majumdar, S. J. Cornell, A. J. Bray, and C. Sire, Phys. Rev. E 56, 2702 (1997).

20 C. Escudero, Ann. Phys. 324, 1796 (2009).

21 C. Escudero, Phys. Rev. Lett. 100, 116101 (2008).

22 C. Escudero, arXiv:0907.0898.

23 C. Escudero, arXiv:1001.3323.
24 H. S. Wio, Int. J. Bif. Chaos 19, 2813 (2009).

25 D. Forster, D. R. Nelson, and M. J. Stephen, Phys. Rev. A 16, 732 (1977).

26 D. S. Jones and B. D. Sleeman, Differential Equations and Mathematical Biology (CRC Press, London, 2003).

27 A. Berera and D. Hochberg, Phys. Rev. Lett. 99, 254501 (2007).

28 A. Berera and D. Hochberg, Nucl. Phys. B 814, 522 (2009).

29 H. S. Wio, J. A. Revelli, R. R. Deza, C. Escudero, and M. S. de la Lama, EPL 89, 40008 (2010).

30 H. S. Wio, J. A. Revelli, R. R. Deza, C. Escudero, and M. S. de la Lama, Phys. Rev. E 81, 066706 (2010).

31 H. S. Wio, C. Escudero, J. A. Revelli, R. R. Deza, and M. S. de la Lama, Philos. T. R. Soc. A (in press). 